\title{
UHF Emission due to Bremsstrahlung from Lightning Return Stroke
}

\author{
Mahendra Singh \\ Department of Physics, Jagdam College, Chapra 841301, India
}

\begin{abstract}
Assuming lightning discharge as plasma it is argued that the high electric field of return stroke produces energetic electrons $(\geq 100 \mathrm{keV}$ ) and colliding energetic particles give rise to Bremsstrahlung which is a possible source of UHF emissions. The computed power of UHF wave for time and frequency responses is reported. In this theoretical study it is shown that a measurable amount of Bremsstrahlung power in UHF or microwave regions is generated by return stroke.
\end{abstract}

Keywords: Bremsstrahlung X-ray, Collision frequency, Lightning return stroke, Stepped leader, Plasma, Thunderstorm

\section{Introduction}

Lightning channels radiate electromagnetic energy covers a wide frequency range extending from a few hertz in the ELF range to beyond the visible region. Rai et al. [1] suggested that Bremsstrahlung might be the operating mechanism for radiation in the UHF or microwave region. Bremsstrahlung is a possible source of UHF or microwave emissions from lightning discharges Rai [2]. The signal strength is high in frequency bands pertaining to VLF $(3-30 \mathrm{kHz})$ and ELF $(3 \mathrm{~Hz}-3 \mathrm{k} \mathrm{Hz})$. This is the cause that researchers have made measurements for these frequency bands. There are relatively few studies on the radiated power in UHF or micro wave regions except the few workers [3 - 7].

Wilson [8] suggested that the strong electric field of thunderclouds could produce electrons with energies up to several MeV. Number of investigators [9-11] attempted to test his suggestion and obtained positive results that energetic electrons are being produced by high electric fields of thunderclouds. Several observational reports [11-13] show that the large electric fields which occur in thunderclouds along with lightning discharges are able to produce $\geq 100 \mathrm{keV}$ electrons.

D'Angelo [14] has argued that electron energization takes place at return stroke wavefronts in a lightning event. When vertical channel of excessive electrons, called a stepped leader, comes near the ground at a distance of 20 to $70 \mathrm{~m}$ above it, a very large electric field of value $10^{7} \mathrm{Vm}^{-1}$ [15] near the ground produces a positively charged return stroke propagating towards the clouds and the leader is effectively connected to the ground potential. The return stroke wavefront carries ground potential upwards, along the partially ionized stepped leader. Since electric field in the return stroke ionizing front is directed upwards, it accelerates electrons downwards. The radius of return stroke channel is of the order of centimeter, while the electron density that the wave front leaves behind the channel is $\sim 2 \times 10^{23} \mathrm{~m}^{-3}[16]$.

Rai [1] has argued that a Bremsstrahlung emission from fully ionized channel of return stroke is negligible. The purpose of this study is to test whether measurable amounts of power in UHF or microwave regions is emitted from the lightning return stroke or not.

In the present study it is shown that the variation of the radiated power with frequency has a Gaussian shape with a maximum value at a certain frequency. Further, it is shown that the lightning return stroke emits an appreciable amount of Bremsstrahlung power in UHF or microwave region.

\section{Total Radiation From Thunderstorm}

Bremsstrahlung is a radiation when velocity of particles changes due to coulomb interaction with particles of the neighborhood. When electrons are distributed uniformly along every direction, they approach the heavy ions from all directions with all possible speeds. This radiation is isotropic, and the polarization is random. Bremmstrahlung in return stroke plasma is the radiation from electrons under the influence of electric field of the nearly positive charges and due to encounters between electrons and atoms. The former is dominant in highly ionized plasmas like return stroke and later in the partially ionized stepped leader. The minimum distance of impact is the de Broglie wavelength of the particles. 


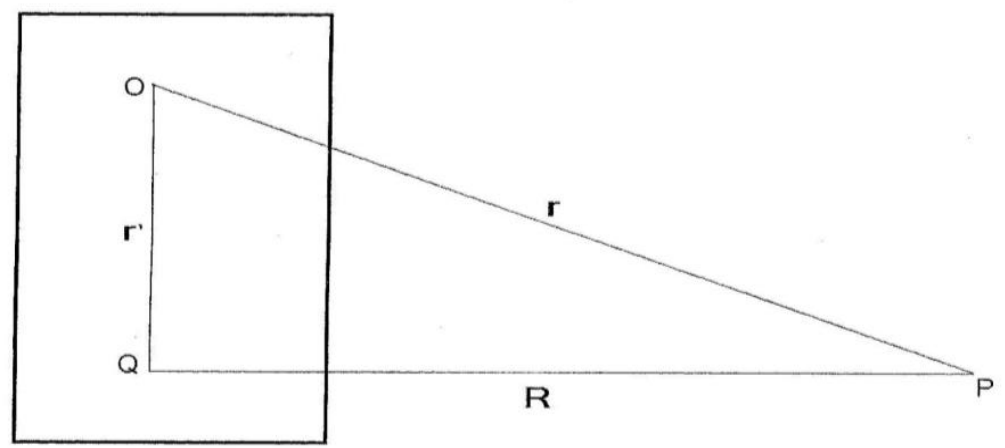

Fig. 1

For far field radiation, the largest length of the source volume is small compared to its distances from the observation point in the radiation zone. If the origin $\mathrm{O}$ is taken within the source volume, then $\mathrm{OP} \approx \mathrm{OQ}$ [Fig.1] and Poynting flux of energy $\mathbf{S}$ radiated from return stroke is written as

$$
\mathbf{S}=\sqrt{\frac{\epsilon_{0}}{\mu_{0}}} \mathrm{E}^{2} \widehat{\mathbf{n}}
$$

Where, $\epsilon_{0}\left(=8.85 \times 10^{-12} \mathrm{Fm}^{-1}\right)$ and $\mu_{0}$ are permittivity and permeability of the free space, respectively, $\widehat{\boldsymbol{n}}$ is a unit vector in the direction of observation, and $E$ is the electric field.

In the far zone, the Poynting energy flows out radially and the field has the character of spherical waves spreading out radially from the accelerated electron.

Classically, an accelerated electron with acceleration $f$ radiates energy. Thus, the total radiation passing through surface area $A$ of a sphere of radius $R$ is

$$
\mathrm{P}=\iint \mathrm{SdA}=\frac{\mathrm{e}^{2}}{6 \pi \epsilon_{0} \mathrm{c}^{3}} \mathrm{f}^{2}
$$

Lightning return stroke is collision dominant plasma. The non relativistic velocity of the energetic electrons in the partially ionized plasma will change according to

$$
\frac{\mathbf{d v}}{\mathbf{d t}}(=\mathrm{f})+v \mathrm{v}=\frac{\mathrm{eE}}{\mathrm{m}}
$$

From (2) and (3), we have

$$
\mathrm{P}=\frac{\mathrm{e}^{4} \mathrm{E}^{2}}{6 \pi \epsilon_{0} \mathrm{~m}^{2} \mathrm{c}^{3}} \mathrm{e}^{-2 v \mathrm{t}}
$$

Where, e $\left(=1.6 \times 10^{-19} \mathrm{C}\right), v$ is the effective collision frequency within return stroke plasma and $\mathrm{m}$ $\left(=9.1 \times 10^{-31} \mathrm{~kg}\right)$ is the mass of electron.

The magnitude of the power of Bremsstrahlung is controlled by the number of electrons accelerated along the return stroke channel and the collision frequency of energetic particles.

The number of electrons, $n_{e}$ accelerated along the return stroke channel of infinitesimally small length $\mathrm{dx}$, is

$$
\mathrm{n}_{\mathrm{e}}=\pi \mathrm{r}^{2} \mathrm{~N}_{\mathrm{e}} \int \mathrm{dx}
$$

Where, $\mathrm{r}$ is the radius of return stroke, $N_{e}$ is the number density of electron, $\mathrm{x}=\int_{0}^{\mathrm{t}} \mathrm{V} \mathrm{dt}$ and $\mathrm{V}$ is the velocity of return stroke.

The return stroke velocity model [17] is written as

$$
V=V_{o}\left(e^{-\mathrm{a} t}-e^{-b t}\right)
$$

Srivastava [15] obtained the following values of $\mathrm{V}_{0}$, a and $\mathrm{b}$

$$
\mathrm{V}_{0}=3.0 \times 10^{8} \mathrm{~ms}^{-1}, \quad a=6.0 \times 10^{4} \mathrm{~s}^{-1}, \quad \mathrm{~b}=7.0 \times 10^{5} \mathrm{~s}^{-1}
$$

From (5) and (6) we have

$$
n_{e}=\pi r^{2} V_{o} N_{e}\left(\frac{e^{-b t}}{b}-\frac{e^{-a t}}{\mathrm{a}}+\frac{1}{\mathrm{a}}-\frac{1}{b}\right)
$$

Now, the total radiation from the lightning return stroke is written as

$$
\mathrm{P}_{\mathrm{T}}=\frac{\mathrm{e}^{4} \mathrm{E}^{2}}{6 \pi \epsilon_{0} \mathrm{~m}^{2} \mathrm{c}^{3}} \mathrm{e}^{-2 v \mathrm{t}} \mathrm{n}_{\mathrm{e}}
$$

Using Eq. (7) in (8), we get

$$
\mathrm{P}_{\mathrm{T}}=\mathrm{P}_{0} \mathrm{e}^{-2 v \mathrm{t}}\left(\frac{\mathrm{e}^{-\mathrm{bt}}}{\mathrm{b}}-\frac{\mathrm{e}^{-\mathrm{at}}}{\mathrm{a}}+\frac{1}{\mathrm{a}}-\frac{1}{\mathrm{~b}}\right)
$$


Where, $\mathrm{P}_{0}=\frac{\mathrm{e}^{4} \mathrm{E}^{2} \mathrm{r}^{2}}{6 \pi \epsilon_{0} \mathrm{~m}^{2} \mathrm{c}^{3}} \mathrm{~V}_{0} \mathrm{~N}_{\mathrm{e}}$.

\section{Frequency Spectrum}

The frequency spectrum of the lightning discharge is obtained from the Fourier transform of the total power of Bremsstrahlung:

$$
\begin{aligned}
P(\omega)= & P_{0} \sqrt{ }\left[\left\{\frac{B}{B^{2}+\omega^{2} b^{2}}-\frac{A}{A^{2}+\omega^{2} a^{2}}+\left(\frac{1}{a}-\frac{1}{b}\right)\left(\frac{v}{v^{2}+\omega^{2}}\right)\right\}^{2}\right. \\
& \left.+\omega^{2}\left\{\frac{a}{A^{2}+\omega^{2} a^{2}}+\frac{b}{B^{2}+\omega^{2} b^{2}}+\left(\frac{1}{a}-\frac{1}{b}\right)\left(\frac{1}{v^{2}+\omega^{2}}\right)\right\}^{2}\right]^{1 / 2}
\end{aligned}
$$

Where, $\omega$ is the frequency in $\mathrm{rad} \mathrm{s}^{-1}$ at which power emission of wave takes place, $A=a^{2}+2 \mathrm{va}$, and $B=b^{2}+$ $2 v b$

\section{Results and Discussions}

Lightning return stroke is a channel of fully ionized plasma, and Bremsstrahlung emission takes place in this plasma due to Coulomb field interaction of electron in its hyperbolic or parabolic orbit around the ion or atom. Electron velocity in the partially ionized channel is non relativistic [2].

With the help of the accepted values for certain parameters associated with return stroke, we compute the radiated power due to the Bremsstrahlung. Thus for the study of the time and frequency responses of power of the Bremsstrahlung emitted from lightning return stroke, the following values [12] have been taken for the parameters used in Eq.(9)

$$
N_{e}=10^{25} \mathrm{~m}^{-3} \quad \mathrm{r}=10^{-2} \mathrm{~m} \quad v=6.4 \times 10^{10} \mathrm{~s}^{-1}
$$

The magnitude of the electric field is taken as $5 \times 10^{5} \mathrm{Vm}^{-1}$ close to the expected break down field in the clouds.

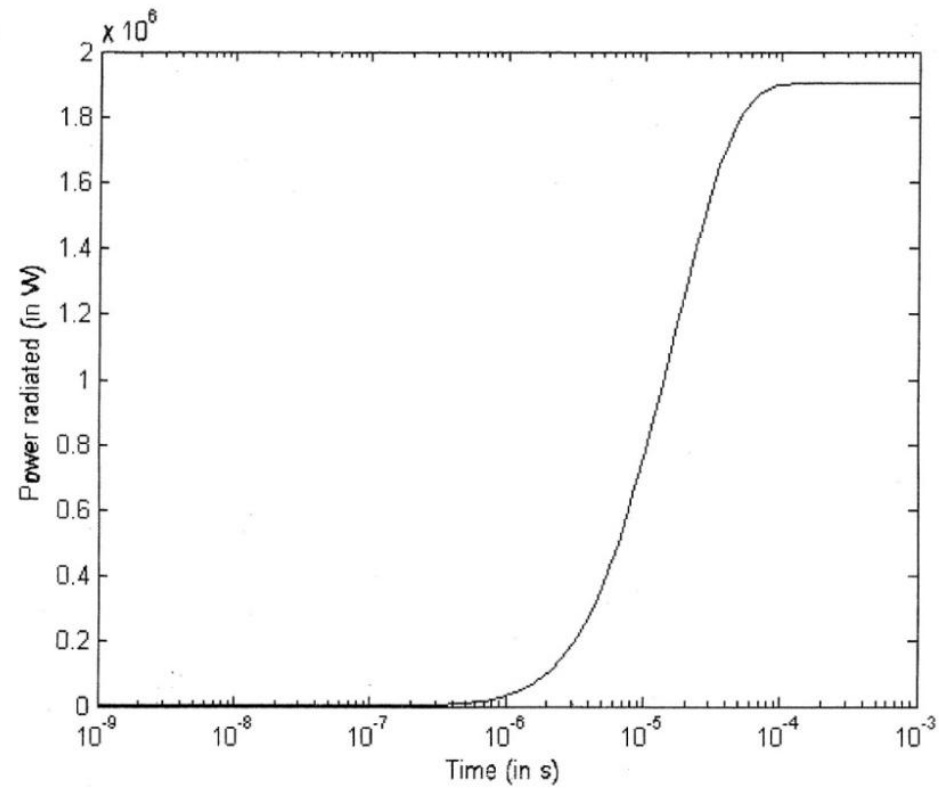

Fig.2 Variation radiated power with time

Variation of the power emitted due to Bremsstrahlung with time is depicted in Fig.2. It is obvious from the figure that the power has the shape as the sign of inclined integration. The radiated power remains almost zero up to $8 \times 10^{-7} \mathrm{~s}$ and thereafter it rises linearly with time. Rai [2] has argued that fully ionized media like return strokes are completely opaque to the UHF radiations. Thus the entire radiation is absorbed within the return channel and one observes no radiation coming out. In contrary to this report it is found that there is an appreciable amount of radiation coming out from return stroke. This is due to increase in number of electrons to be energized to produce Bremsstrahlung. After $10^{-4} \mathrm{~s}$, when channel volume gets increased, the contribution to the Bremsstrahlung power comes out to be its saturation value of $1.9 \times 10^{6} \mathrm{~W}$.

The spectral characteristics of the radiated power of Bremsstrahlung is computed by using Eq.(10) and its variation with frequency is shown in Fig. 3. The variation of power with frequency has a Gaussian shape with a peak value $7.13 \times 10^{-5} \mathrm{WHz}^{-1}$ at frequency $2.5 \times 10^{10} \mathrm{~Hz}$. It is obvious from this figure that a measurable amount of Bremsstrahlung power in UHF or microwave regions is produced by lightning return stroke. 


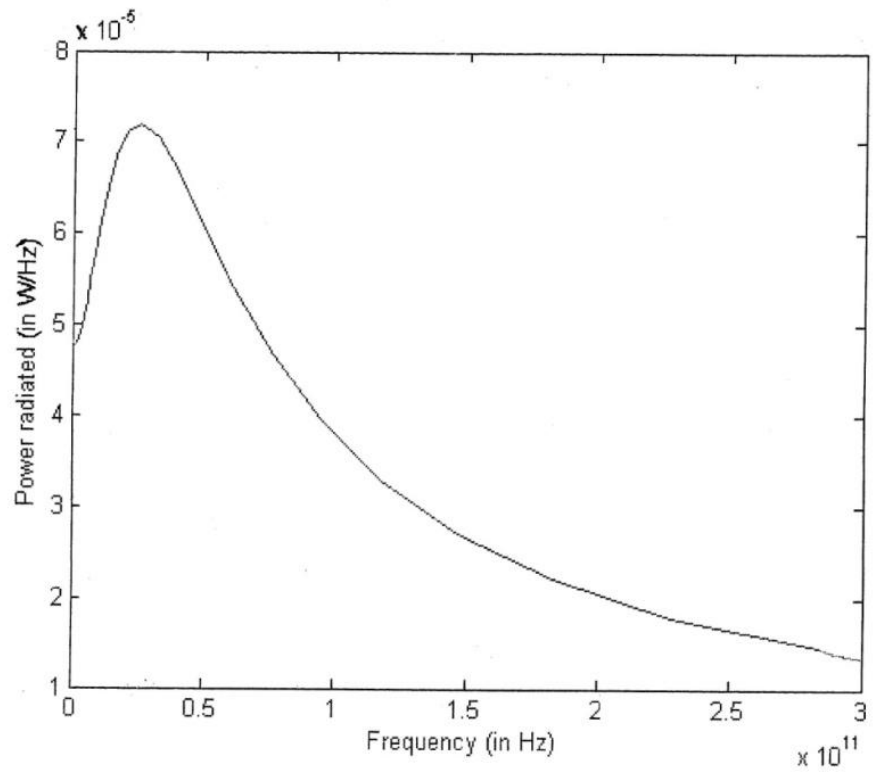

Fig. 3 Variation of radiated power with frequency

\section{Conclusions}

The large electrostatic fields due to charge imbalance during lightning process are able to produce energetic electrons with energies up to $\geq 100 \mathrm{keV}$. Appreciable amounts of Bremsstrahlung power in UHF regions are generated by these electrons.

\section{References}

[1]. J. Rai, M. Rao, and B. A. P. Tantry, Bremsstrahlung as a possible source of UHF emission from lightning, Nature, 238, 1972, 5960 .

[2]. J. Rai, On the origin UHF of atmospherics, J Atmos Terr Phys, 40, 1976, 475-478.

[3]. M. Takagi, Takeuti, Atmospheric radiation from lightning discharge. Proc Res Inst Atmos, 10, 1963, 1-11.

[4]. M. Brook and N. Kitagawa, Radiation from lightning discharges in the frequency range 400 to $1000 \mathrm{M}$ c/s, J Geophys Res, 69 , 1964, 2431-2434.

[5]. E. L. Kosarev, V. G. Zatsepin and A. V. Mitrafanov, Ultrahigh frequency radiation from lightning, J Geophys Res ,75, 1970, 75247530 .

[6]. Devendraa Siingh, R. P. Singh, Ashok K. Singh, Sanjay Kumar, M. N. Kulkarni and Abhay K. Singh, Discharge in the Stratosphere / Mesosphere, Spac Sci Rev, 169,2012, 73-121.

[7]. Devendra Siingh, A. K, Singh, R. P. Patel, R. Singh, R. P. Singh, B. Vennadhari, and M. Mukherjee, Thunderstorm, lightning, sprites and magnetospheric whistler - mode waves,Survey in Geophys, 29, 2008, 499 - 551.

[8]. C. T. R. Wilson, The acceleration of $\beta$ - particles in strong electric fields such as those of thunder clouds, Proc Cambridge Phil Soc, $22,1924,534-538$

[9]. B. F. J. Schonland, and J. P. T. Viljoen, On a penetrating radiation from thunder clouds, Proc Roy Soc London, A140, 1933, 314333.

[10]. D. P Whitemire, Search for high energy radiation near lightning strokes, Lett. Nuovo Cimento, 26, $1979,497-501$.

[11]. G. K. Parks, B. H. Mauk, R. Spiger and J. Chin, X-rays enhancements detected during thunderstorm and lightning activities, Geophys Res Lett, 8, 1981, 1176-1179.

[12]. M. McCarthy, and G. K. Parks, Further observations of X-rays inside thunderstorms, Geophys Res Lett, 12, 1985, 393-396.

[13]. W. A. Macky, An attempt to detect radiation in thunderclouds, Proc Cambridge Phil Soc, 30, 1934, 70-73.

[14]. N. D'Angelo, On X-rays from thunderclouds, Annales Geophysicae, 5B, 1987, 119-122.

[15]. M. A. Uman, Lightning (Dover Inc New York, 1984).

[16]. M. A. Uman and R. E. Orville, Electron density measurement in lightning from stark-broadening of Ha, J Geophys Res, 69, 1964, 5151-5154.

[17]. K. M. L. Srivastava, Return stroke velocity of a lightning discharge, J Geophys Res, 71, 1966, $1283-1286$. 